\title{
La dernière ouvre de Christian Knorr de Rosenroth : le Messias puer
}

Par

\section{Anna Maria Vileno}

FNRS - ULB, Bruxelles

\section{Robert J. Wilkinson}

Valley House, Temple Cloud, Somerset

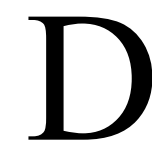
ans un des rares articles qu'il a consacrés à la kabbale chrétienne, Gershom Scholem identifie trois auteurs chrétiens qui se distinguent selon lui par leur intérêt sincère pour la tradition ésotérique du judaïsme. ${ }^{1}$ Après la génération de Johannes Reuchlin (1455-1522) dont il aurait pu se croire être la réincarnation ${ }^{2}$, Scholem cite Guillaume Postel (1510-1581), Christian Knorr de Rosenroth (1636-1689) et Franz Joseph Molitor (17791860). Dans une perspective teintée de romantisme, ce dernier considérait la tradition kabbalistique comme une puissance souterraine capable de vivifier à la fois le judaïsme et le christianisme, conception qui a exercé une forte influence sur la manière dont Scholem lui-même a

\footnotetext{
${ }^{1}$ SCHOLEM 1988a, p. 198.

${ }^{2}$ « Si, compte-tenu de l'état actuel de la recherche sur la kabbale, je croyais à la métempsychose, je m'imaginerais être une sorte de réincarnation de Jean Reuchlin qui fut le premier non-juif à étudier la langue et le monde du judaïsme, et qui inaugura, voilà près de cinq cents ans, la science du judaïsme en Europe.» (SCHOLEM 1988a, p. 191).
} 
envisagé la mystique juive dans le cadre de l'histoire générale du judaïsme. ${ }^{3}$ Guillaume Postel a le mérite d'avoir donné une traduction, certes très personnelle, du Zohar avant même que le texte original ne paraisse en version imprimée.

Le deuxième auteur évoqué est généralement moins bien connu, de même que le rapport que Scholem entretenait avec lui. Christian Knorr de Rosenroth est un kabbaliste chrétien allemand qui s'est illustré par la traduction latine de textes appartenant principalement à la tradition lourianique et compilés dans l'anthologie intitulée Kabbala denudata, publiée entre 1677 et $1684 .^{4}$ La qualité de son travail de traducteur n'a pas échappé à Scholem, de même que la fiabilité de ses sources hébraïques. ${ }^{5}$ Lorsqu'elle est possible, la comparaison de la version hébraïque et de la traduction de Knorr révèle que cette dernière est fidèle et conforme au texte original. Par ailleurs, Knorr démontre une volonté de comprendre en profondeur la polysémie des textes kabbalistiques, comme on peut le voir dans ses Loci communes kabbalistici ${ }^{6}$, lexique des notions kabbalistiques les plus fréquemment usitées et dont chaque entrée expose les différentes interprétations envisageables. Le texte n'est ainsi jamais réduit à une unique lecture.

En dehors des traductions et des échanges épistolaires avec les personnalités de son temps ${ }^{7}$, Knorr a aussi fait activement œuvre de kabbaliste chrétien. Certains travaux, tel l'Adumbratio kabbalce christiance ${ }^{8}$ probablement rédigé de concert avec son comparse de toujours, l'alchimiste belge François-Mercure van Helmont et publié en 1684 dans le second tome de la Kabbala denudata, reflète son investissement dans une réflexion qui vise à rapprocher la doctrine chrétienne des enseignements kabbalistiques. ${ }^{9}$ Mais en dehors de la production propre et originale que constitue l'Adumbratio, Knorr est également l'auteur d'une édition du Zohar ainsi que d'une nouvelle édition du Nouveau Testament syriaque, publiée en alphabet hébraïque carré non vocalisé à Sulzbach en $1684 .{ }^{10}$ L'année 1684 constitue une année importante dans le processus de maturation intellectuelle chez Knorr et avec cette Peshitta, notre auteur pose un acte important dans l'économie générale de la kabbale chrétienne. ${ }^{11}$ Cependant, Christian Knorr de Rosenroth est également l'auteur d'un texte intitulé Messias puer qui semble représenter l'aboutissement de ses recherches kabbalistiques dans une perspective

\footnotetext{
${ }^{3}$ Scholem s'est lui-même exprimé au sujet de cette influence dans la lettre qu'il adresse à son éditeur Zalman Schoken, en 1937 et traduite en français dans SCHOLEM 1988b, p. 7-9. Sur l'influence de Molitor sur Scholem, voir notamment BIALE 2001, p. 66-67.

${ }^{4}$ La proportion de kabbale lourianique contenue dans la Kabbala denudata est l'objet de nombreuses discussions. Pour notre part, nous estimons qu'un peu plus de cinquante pourcents de l'anthologie relèvent de la tradition lourianique.

${ }^{5}$ Scholem s'est particulièrement intéressé à un ouvrage assez mystérieux, mêlant alchimie et kabbale dont Knorr a donné la traduction, morcelée à travers les Loci communes kabbalistici, et qui constitue la seule attestation du texte qui nous soit conservée. Scholem concluait à l'existence d'un original hébraïque ayant servi de base à Knorr. Cf. SCHOLEM 1990, p. 132 sq.

${ }^{6}$ KNORR DE ROSENROTH 1677, p. 1-740.

${ }^{7}$ La correspondance entre Knorr et Henry More a été publiée dans KNORR DE RosenROTH 1677, «Pars secunda ».

${ }^{8}$ L'ouvrage a fait l'objet d'une traduction récente : ROUSSE-LACORDAIRE 2018.

${ }^{9} \mathrm{Il}$ a été longuement discuté ailleurs la manière dont il convient de concevoir le rapport entre kabbale et christianisme dans la pensée de Knorr : VILENO 2016.

${ }^{10}$ KNORR DE ROSENROTH 1684a.

${ }^{11}$ Nous avons donné une description de cette Peshitta et de son importance dans l'œuvre de Knorr dans un article à paraître dans le prochain volume de Morgen-Glantz, vol. 28, 2018.
} 
chrétienne, texte considéré jusqu'à présent comme perdu par l'ensemble de la communauté scientifique.

\section{Les traces du manuscrit perdu}

Dans une étude consacrée à l'amitié qui liait Knorr de Rosenroth à Leibniz, Allison Coudert mentionne une lettre de ce dernier adressée à Gehrardt Molanus (1633-1722), théologien luthérien allemand dans laquelle Leibniz dit: "Knorr a achevé un livre remarquable intitulé Messias puer dans lequel l'histoire du Christ depuis sa conception jusqu'à la disputation au Temple est très joliment illustrée par un grand nombre de passages extraits des kabbalistes. $»^{12}$

Dans l'ouvrage qu'elle dédiait à l'étude des rapports entre Leibniz et la pensée kabbalistique, Coudert rapporte une autre lettre adressée cette fois par le philosophe à Hiob Ludolph (1624-1704), orientaliste et auteur d'une histoire de l'Éthiopie ${ }^{13}$ à qui Leibniz recommande vivement les travaux de Knorr. Il y décrit à nouveau le Messias puer: "Il m'a montré une ouvre pratiquement achevée dont le titre est Messias puer, dans laquelle l'histoire du Christ depuis l'annonciation jusqu'au baptême, telle qu'elle est rapportée par les Evangélistes et d'autres [auteurs], est admirablement illustrée par d'anciens passages kabbalistiques. $\gg^{14}$

Il est bien connu que Leibniz appréciait au plus haut point les travaux de Knorr et il a, semble-t-il, fait part de son enthousiasme au sujet du Messias puer, dans une lettre à Christoph Daniel Findekeller (1634-1694). Si le courrier de Leibniz, daté du 16/26 janvier 1688, est aujourd'hui perdu, nous conservons la réponse que lui envoie Findekeller en février de la même année: "Pour ce qui est de Mr Knorr de Rosenroth, Directeur de la Chancelerie à Sulzbacq, il y a long temps, que j'ay de l'honneur de le connoistre par la voye de mon beaufrere, Glimper, qui lui avoit procuré l'amitié de feu $M^{r}$ le Baron de Frisen; ce que vous me marquez de lui et de son manuscrit du Messias Puer, sera quelque chose de curieux, quand il le mettra aujour, mais possible que ce Mss ${ }^{t}$ ne restera dans la Bibliotheque de son Prince. $\rangle^{15}$

Un peu plus loin, Coudert signalait que le manuscrit du Messias puer semble avoir été perdu. ${ }^{16}$ La question se pose dès lors de savoir ce qu'il est advenu de ce manuscrit que

\footnotetext{
12 «Absolvit Knorrius elegans opus cui titulum fecit, Messias Puer, in quo Christi Historia inde a conceptu usque ad disputationem in templo collatis Cabbalistarum locis pulcherrime ornatur. » (DEUTSCHE AKADEMIE DER WiSSENSCHAFTEN 1923, Lettre du 24 (?) avril 1688, I, 5, 109 cité dans COUDERT 1996, p. 470).

${ }^{13}$ LUDOLF 1681.

14 «Ostendit mihi jam prope confectum opus, cui titulus est: Messias puer, ubi Historia Christi ab annunciatione ad baptismum usque, quae ab Evangelistis traditur cum aliunde, tum ex locis veterum cabbalistarum mirifice illustratur. " (DEUTSCHE AKADEMIE DER WISSENSCHAFTEN 1923, Lettre 30 April/9 September 1688 cité dans COUDERT 1995, p. 169).

${ }^{15}$ Deutsche AKAdemie Der Wissenschaften 1954, 1, 5, p. 55. La note de bas de page de l'éditeur de la correspondance de Leibniz mérite d'être rapportée : «Knorr Messias puer ist nie erschienen. Nach Knorrs Tod besaß die Handschr. Fr. Merkur van Helmont, der das Werk in Amsterdam drucken lassen wollte (K. Salecker Chr. Knorr von Rosenroth 1931 S. VIII und 12f.); Prince: Pfalzgraf Christian August von Sulzbach ».

16 " The next two entries in the journal dealing with van Helmont concern Leibniz's role in the publication of van Helmont's and von Rosenroth's works. In the first, Leibniz refers to the negotiations he had undertaken at the request of van Helmont involving the publication of the second edition of van Helmont's and von Rosenroth's translation of Boethius' The Consolation of Philosophy. This appeared in Lüneburg in 1697 and Leibniz wrote the preface for it. The second describes his involvement with two works of von Rosenroth, The Harmony of the
} 
Leibniz décrit par deux fois avec enthousiasme et qu'il avait vu lors de la visite rendue à Christian Knorr. ${ }^{17}$ Une piste semble être que Knorr ait confié le manuscrit à François-Mercure van Helmont afin qu'il le fasse imprimer à Amsterdam. Ainsi, Coudert cite une missive de van Helmont à Leibniz, alors bibliothécaire en charge de la bibliothèque du Duc Anton Ulric de Brunswick, dans laquelle van Helmont évoque la possibilité de faire imprimer à Amsterdam certains ouvrages de Knorr de Rosenroth dont on lui demande des copies. ${ }^{18}$ C'est vraisemblablement la piste suivie par le rédacteur de la notice de l'Encyclopedia universalis consacrée à Christian Knorr de Rosenroth : «Au soir de son existence, Knorr voulut écrire un livre sur l'enfance de Jésus, fondé sur les sources rabbiniques et kabbalistiques. Il en envoya le manuscrit à son ami Van Helmont, qui promit de le faire publier à Amsterdam. Le projet cependant ne vit jamais le jour, et cette ouvre importante, Messias Puer, fut perdue. ${ }^{19}$

\section{Un manuscrit conservé à la HAB}

Le catalogue de la Herzog August Bibliothek de Wolfenbüttel (HAB) recense sous le nom de Knorr un codex portant le titre Historice Evangelica initium, non daté dans le catalogue. Le titre complet a été ajouté par la main de Knorr lui-même, au-dessus de la première page de texte: Historice evangelica initium, secundum quatuor Evangelistas ${ }^{20}$. Nous savons que le texte est complet, puisqu'il porte la mention «Finis» au folio 52v. Il existe une version partiellement imprimée du même texte, conservée à la Bayerische Staatsbibliothek de Munich (BSB). Comprenant une centaine de pages, la version imprimée s'interrompt après la première moitié du folio 19r, reproduisant ainsi un peu moins d'un tiers du texte total du manuscrit. La dernière page imprimée comporte une note manuscrite indiquant que « l'impression n'a pas été poursuivie en raison de la mort de l'auteur $»{ }^{21}$ Le catalogue de la BSB, s'il ne donne pas de lieu d'impression de l'œuvre, la date de 1690. Pourtant, l'année 1689 est plus vraisemblable, compte-tenu du fait que Knorr décède en avril 1689 et que le processus d'impression semble avoir pris fin avec sa mort.

Le manuscrit est un in-octavo, conservé dans une demi-reliure à coins en basane, de couleur brune, datant de la fin du $17^{\mathrm{e}}$ siècle, et qui constitue probablement la reliure originale. Hormis une page de garde, le codex comprend 52 folios recto-verso, soit 104 pages. Le papier apparait bruni, mais le texte est lisible. Plusieurs rédacteurs sont intervenus sur le texte, mais

Evangelists, which was published, and the manuscript of Messias Puer, which appears to have been lost.» (COUDERT 1995, p. 64) (c'est nous qui soulignons).

${ }^{17}$ Coudert revient abondamment sur le long séjour que Leibniz effectue à Sulzbach du 31 décembre 1687 au $1^{\text {er }}$ février 1688, elle en fait même le cœur de son argumentation visant à démontrer l'intérêt et la profonde implication de Leibniz dans les recherches kabbalistiques de François-Mercure van Helmont et de Christian Knorr de Rosenroth. Cf. COUDERT 1995, passim.

${ }^{18}$ «Les escripts de Mr. Knor de Rosenrot, qui S.A. Serme. Monseigr. le Duc Antoine Ulric de Bronsvic demande, pur le faire imprimer. je iuge a propos comme en peu de jours ispere daller a Amsterdam de les prendre avecq moy pour voir si La ie les peu mieux faire imprimer quoy faisant Vous aures part de mesme ie donnerey a S. A Le Serme. Je demeure Comme Vous saves, Monsieur. » (LBr 389, f. 49, Ter Borg, October, 1696, cité dans Ibid., p. 181).

${ }^{19}$ GAREL 2018.

${ }^{20}$ Cod. Guelf. 126. Extrav. folio 1r.

${ }^{21}$ «Plura non sunt impressa. Auctor operi est immortuus. » (Historice evangelicce initium, BSB, p. 96). 
l'écriture principale est de type humanistique, elle appartient à des secrétaires ${ }^{22}$, des scribes professionnels que Knorr avait à sa disposition en tant que fonctionnaire de la cour de Sulzbach. Le texte apparaît comme une mise au propre ayant de toute évidence servi de base au travail de l'imprimeur et représente donc une version relativement aboutie du processus de rédaction. Pourtant, même à ce stade, le texte a encore été profondément remanié, comme en témoignent les nombreux ajouts, corrections et suppressions qui apparaissent en marge, tous autographes de Knorr. La plupart des corrections ont été intégrées dans la version imprimée et conservée à la BSB. ${ }^{23}$

Se développant sous la forme d'un dialogue entre deux personnages, "Christianus » et «Cabbalista Catechumenus », le texte consiste en un commentaire suivi, verset par verset, du débuts des évangiles de Luc, de Matthieu et de Jean, introduits dans la version syriaque du Nouveau Testament éditée par Christian Knorr de Rosenroth en $1684^{24}$. Le texte syriaque de l'Évangile commenté figure à l'ouverture de chaque section sur de petits feuillets, collés sur la moitié gauche de la page. La version syriaque est accompagnée de la traduction latine des versets concernés ${ }^{25}$, réalisée par Knorr au départ de la version syriaque et divergeant par conséquent à de nombreuses reprises de la Vulgate. Le texte est divisé en dix sections. Ainsi, ce sont les deux premiers chapitres de l'évangile de Luc, les cinq premiers versets du premier chapitre de Jean et les deux premiers chapitres de l'évangile de Matthieu qui sont commentés de façon extrêmement détaillée par les deux personnages. L'exégèse fait appel non seulement à la littérature kabbalistique, notamment zoharique et lourianique, mais aussi à la littérature talmudique, midrashique, aux targumim et à la littérature juive pieuse contemporaine de Knorr.

Le codex ne présente pas de page de titre, le texte débute au premier folio numéroté, le titre ayant été ajouté a posteriori. Or, le titre tel qu'il apparaît dans l'ajout et dans les catalogues Historice evangelica initium - n'est mentionné dans aucune source contemporaine ou ultérieure à Knorr. En revanche, Messias puer figure régulièrement au nombre des œuvres connues de l'auteur, comme l'atteste par exemple une notice de Pierre Bayle au sujet de Knorr, "cet excellent homme qui nous a donné la Cabbala Denudata, \& qui travaille presentement à l'Ouvrage dont voici le titre : Messias Puer, Ex Antiquitatibus Hebrceorum, \& in specie è Libro SOHAR ad Textum N.T. Syriacum illustrans cum Sesqui-Centuria Locorum, Textibus N.T. variè paralellorum, excerptum è Libro SOHAR cum Textu originario, \& Versione Latina Opusculum, In gratiam convertendorum Judceorum $\kappa \alpha \tau^{\prime}{ }^{\alpha} v \theta \rho \omega \pi o v$ [ad hominem] conscriptum. $»^{26}$

\footnotetext{
${ }^{22}$ Un premier secrétaire a rédigé la plus grande partie du texte. Knorr prend la relève le temps d'une demi page au folio 49r et un second secrétaire intervient à partir de la seconde moitié du folio 49r. Quelques lignes sont de la main de Knorr au folio 50r, ainsi que les quatre derniers folios (51r-v, 52r-v).

${ }^{23}$ Le codex Guelf. 126. Extrav. ne figure pas dans la description du fonds Knorr présentée par Rosmarie Zeller dans ZELLER 2006, qui s'attache principalement à la correspondance de Knorr. De plus, le corps de texte n'est pas de la main de Knorr lui-même. En revanche, le codex est mentionné dans ARNOLD 2009, p. 65.

${ }^{24}$ Cf. supra note 10.

${ }^{25}$ Soulignée dans le texte, la traduction latine apparaît en italique dans la version imprimée de la BSB.

${ }^{26}$ BAYLE 1687, p. 332.
} 
De même, certaines notices d'encyclopédies ultérieures font état du titre Messias puer ${ }^{27}$. Dans l'édition de 1740, Le grand dictionnaire historique de Louis Moreri précise que le Messias puer « se trouve encore en manuscrit ${ }^{28}$

Non seulement, le titre Messias puer est clairement associé à Christian Knorr de Rosenroth, mais de plus, l'analyse du contenu de l'Historice evangelicae initium correspond de façon précise à la description de l'œuvre telle qu'elle se rencontre dans la correspondance de Leibniz $^{29}$. Le commentaire porte en effet sur les parties des évangiles décrivant la conception du Christ et l'annonce de sa naissance - avec une large attention accordée aux figures de Jean et de ses parents, Zacharie et Elisabeth -, sa naissance proprement dite, son enfance et son adolescence. La dernière section du texte est intitulée «De Sapientia Jesu duodecennis in Templo demonstrata. ê Luc. 2, v. 40-52 ». ${ }^{30}$

\section{Les Parallelismi Soharitici}

Dans la préface au second tome de la Kabbala denudata, Knorr annonce la parution prochaine, Deo favente, d'un nouvel ouvrage : "En outre, j'ai ajouté à l'adresse de ceux pour qui il est plaisant de citer les dits du Zohar, soit dans les discussions, soit encore dans des écrits, une plus longue liste des parallèles utilisés [entre le Zohar et] les dits du Nouveau Testament, liste que je publierai, avec l'aide de Dieu, en un autre temps. ${ }^{31}$

La même année, à la fin de la courte préface qu'il place à l'ouverture de son édition du Zohar, Knorr annonce «que si Dieu le permet, [il publiera] prochainement les Parallèles entre le Zohar et le Nouveau Testament. $»^{32}$

Étrangement, nous n'avons jusqu'à présent répertorié aucune mention des Parallèles de Knorr dans la littérature des dix-septième et dix-huitième siècles. En revanche, le texte intitulé Historice evangelica initium comprend de très nombreux renvois vers un texte de Knorr lui-même, généralement identifié par le titre Excerpta. Comme indiqué ci-dessus, le manuscrit conservé à la HAB révèle qu'un très grand nombre de corrections ont été apportées par Knorr et ce en dépit du fait qu'il s'agissait déjà d'une version aboutie. Une analyse minutieuse des corrections révèle la grande indécision de Knorr tout au long du texte. Il exerce parfois une importante autocensure sur son travail dont il conviendra d'expliquer les raisons par la suite. Une des corrections concerne précisément le titre de l'ouvrage intitulé Excerpta qui apparaît à deux reprises, dans une version longue : «Loca autem nonnulla $\hat{e}$

\footnotetext{
${ }^{27}$ Guillaume van Gemert a identifié deux encyclopédies néerlandaises qui recensent le Messias puer aux côtés de la Kabbala denudata comme œuvres dont Knorr de Rosenroth est l'auteur. Cf. VAN GEMERT 1991.

${ }^{28}$ MORERI 1740, entrée « Knorr de Rosenroth ».

${ }^{29}$ Cf. supra, notes 12 et 14 .

${ }^{30}$ Aux quatre dernières sections du texte ont été ajoutés des titres explicites : Section 7, « De Nativitate \& Circumcisione Johannis \& Restituto Zachariæ, è Luca, v. 57-80»; Section 8, « De Nativitate, Circumcisione \& Oblatione Christi, è Luc. II, 1-39»; Section 9, « De Adventu Magorum \& Fuga Christi : ex Matth. 2, 1-23»; Section 10, «De Sapientia Jesu duodecennis in Templo demonstrata. ê Luc. 2, v. 40-52 ».

31 «Adjunxi autem, ita in gratiam illorum quibus allegare dicta Soharis voluptas erit, sive in Concionibus sive in scriptis aliis, parallelismum aliqualem dictorum quorundam Novi Testamenti, cujus usum alio tempore, Deo favente, exhibebo uberiorem. » (KNORR DE ROSENROTH 1684b, « Lectori philebræo salutem! », p. 17).

32 «\& si Dominus permiserit Parallelismos Soharisticos in N.T quàm proximè expectet. » (KNORR DE ROSENROTH 1684c, « Lectori benevolo salutem ! »).
} 
Libro Sohar, ubi de utroque Messia agitur, evolvi possunt in Parallelismis atque Excerptis Sohariticis ad Nov. Testamentum. Cent. 1. Loc. 62. usque ad 91. ${ }^{33}$

Le titre Parallelismis atque Excerptis Sohariticis ad Nov[um] Testamentum a été supprimé et remplacé en marge par le seul Excerptis. Or, un ouvrage intitulé «Parallèles entre des extraits du Zohar et le Nouveau Testament »-dont on ne connaît, à ce jour aucun exemplaire - correspond à l'ouvrage annoncé par deux fois par Knorr au cours de l'année 1684. À noter également que ces " Parallèles » semblent être ceux annoncés dans la notice de Pierre Bayle, de l'année $1687^{34}$ à propos d'un ouvrage composé d' "excerptum è Libro SOHAR » du Zohar, ces « extraits » n'étant probablement pas autre chose que les Excerpta auquel l'Historice evangelica initium renvoie de façon répétée.

Les titres de Messias puer et de Parallelismi - ou Excerpta - sont donc intrinsèquement liés, que ce soit à travers la notice de Pierre Bayle ou encore à travers ce que l'on peut observer dans le texte de l'Historice evangelica initium. L'état actuel de nos recherches nous amène à formuler les hypothèses suivantes : tout d'abord, le texte inconnu catalogué sous le titre de Historice evangelica initium est le texte du Messias puer qui ne doit donc plus être considéré comme perdu; ensuite, les Excerpta auquel le Messias puer fait référence sont les "Parallèles entre le Zohar et le Nouveau Testament» qui doivent, eux, être à ce jour considérés comme perdus ; enfin, nous postulons que peu après janvier 1687 (date de la notice de Bayle), Knorr a pris conscience de la difficulté de faire paraître un ouvrage qui intégrât à la fois la présentation des parallèles qu'il avait relevés entre le texte du Zohar et celui du Nouveau Testament et à la fois les exégèses du Nouveau Testament syriaque développées non seulement sur base des principes kabbalistiques, mais reposant également sur la littérature talmudique et midrashique. Dès lors, Knorr a pris la décision de scinder l'ouvrage en deux parties : les matériaux kabbalistiques étaient destinés à prendre place dans un ouvrage intitulé Parallelismi ou Excerpta, tandis que l'exposition de la Peshitta a été réservée pour l'ouvrage intitulé Messias puer et demeuré très vite occulté par son non-titre de Historice evangelicae initium. Dès lors, le Messias puer et les Parallelismi doivent être considérés comme deux ouvrages distincts, certes, mais constituant les deux faces d'une même médaille. Ainsi, la documentation rassemblée dans les Parallèles est déployée à un niveau exégétique dans le Messias puer.

\section{Du Messias puer à l'Historiæ evangelicæ initium}

A priori, l'état du manuscrit conservé à la $\mathrm{HAB}$ ne permet pas de le considérer comme la base de la version imprimée détenue par la BSB. En effet, il est difficilement concevable que l'imprimeur, qui travaille normalement de manière mécanique et rapide, ait pu intégrer autant de corrections et d'ajouts. Toutefois, il apparaît que la plupart des corrections présentes sur le manuscrit ont bel et bien été introduites dans la version imprimée. Deux hypothèses sont alors possibles : soit il existait un manuscrit intermédiaire, "propre », utilisé par l'imprimeur ; soit le manuscrit de la $\mathrm{HAB}$ a pu être directement utilisé si l'on considère que l'auteur, Knorr de Rosenroth, a activement participé au processus d'impression. Cette dernière hypothèse doit probablement être préférée pour plusieurs raisons : tout d'abord, c'est ce que suggère la note

\footnotetext{
${ }^{33}$ KNORR 1689, folio 25r. Une autre mention apparaît au folio 25v, également corrigée : « vide autem de his latius in Parallelismis \& Excerptis Soharisticis Cent. 1. Loc. 54. n. 40.41. » remplacée par « Vide in Excerptis Cent. 1. Loc. 54. n. 40.41.».

${ }^{34}$ Cf. supra, note 26.
} 
manuscrite qui clôt la version $\mathrm{BSB}^{35}$, qui indique clairement une participation active de l'auteur, sans laquelle l'impression n'a pas pu être poursuivie ; ensuite, la collaboration des auteurs à l'impression de leurs propres ouvrages, en particulier dans les domaines des hebraica, est bien documentée ${ }^{36}$; de plus, tous les ajouts et les corrections qui figurent sur le manuscrit ont été intégrés dans la version imprimée ; enfin, ajoutons que la première page comprend une remarque de la main de l'auteur, en allemand, indiquant que «tout ce qui est souligné doit être composé (imprimé) en plus petit [caractère] ${ }^{37}$, ce qui plaide en faveur d'une copie ayant effectivement servi de base à l'imprimeur. Enfin, si l'on considère que l'imprimeur était bel et bien face au manuscrit, cela permet d'expliquer la présence en marge du texte d'une numérotation des feuillets et des cahiers, tracée au crayon rouge et correspondant à la pagination et à la reliure du fragment imprimé. Ces annotations en marge cessent d'apparaître dans le manuscrit à l'endroit où la version imprimée s'arrête également, à savoir au folio $19 \mathrm{r}$.

Dès lors que l'on compare les annotations relatives à la numérotation des feuillets et des cahiers telle qu'elle apparaît dans le manuscrit avec la pagination et la numérotation réelle des cahiers du fragment imprimé, on constate qu'elles ne correspondent pas. La comparaison suggère que deux pages ont été omises au début du manuscrit. En effet, le manuscrit débute avec l'annotation $3 \mathrm{~A} / 1$ et le fragment imprimé avec $\mathrm{A} / 1$, ce qui indique probablement l'omission d'une page de titre. Une telle omission ou suppression de la page de titre a eu pour conséquence que l'œuvre a été nommée, de façon conventionnelle, d'après les premiers mots du texte, en l'occurrence Historice evangelica initium. Ceci explique que les catalogues aient retenu ce titre, plutôt que celui initialement prévu par Knorr de Messias puer.

\section{Conclusion}

Le Messias puer est demeuré à l'état de manuscrit ${ }^{38}$ et bien qu'une version partielle ait été imprimée, il ne semble pas avoir retenu l'attention, ni des contemporains de Knorr, ni des chercheurs ultérieurs. Pourtant, l'œuvre constitue un état d'aboutissement considérable du travail de Knorr en tant que kabbaliste chrétien. En dehors de l'Adumbratio kabbaloe christiance, peu de productions propres de Knorr nous sont connues, si bien que Knorr est considéré avant tout comme un traducteur, un compilateur et un éditeur de textes hébraïques. Pourtant, le Messias puer constitue probablement une des applications les plus rigoureuses des enseignements de la kabbale aux textes du Nouveau Testament. De même que pour l'Adumbratio, la question se pose de savoir à quel type de christianisme Knorr confronte son lecteur. Plusieurs points fondamentaux de la doctrine chrétienne y sont en effet considérablement malmenés et la recherche future devra prendre la mesure de l'écart introduit par Knorr par rapport à la lecture traditionnelle des Évangiles. La conception du Christ par la Vierge fait l'objet de longs développements qui mettent volontiers l'accent sur l'aspect

\footnotetext{
${ }^{35}$ Cf. supra, note 21.

${ }^{36}$ BURNETT 2000, p. 28. L'existence d'une presse hébraïque à Sulzbach est bien attestée, Christian Knorr de Rosenroth et François-Mercure van Helmont ont considérablement contribué à son activité. Dès lors, l'implication de Knorr dans le processus d'impression ne saurait surprendre.

37 «NB. Alles was unterstrichen ist, soll mit kleinerer Schrift gesetzt werd(en)» (KNORR DE ROSENROTH 1689, folio 1r).

${ }^{38}$ Une édition du manuscrit et de toutes ses corrections, ainsi qu'une traduction anglaise largement annotée est actuellement en préparation par nos soins et devrait paraître prochainement.
} 
matériel de cette conception. ${ }^{39}$ De même, la doctrine kabbalistique du gilgul ha-nafshot, de la " révolution des âmes » est abondamment utilisée afin d'interpréter les actes et les pensées des personnages que le texte passe en revue. De même que dans l'Adumbratio, l'Adam Kadmon y occupe une place importante, bien que différente. Enfin, l'ensemble des spéculations relatives au monde divin sont envisagées à travers le filtre des sefirot, le Messias puer postulant la «nature séfirotique » de Dieu. Le commentaire développé dans le Messias puer laisse apparaître une herméneutique audacieuse, voire radicale, fondée sur une relecture complexe mais systématique et cohérente des Évangiles.

\section{ABSTRACT}

Christian Knorr de Rosenroth est l'auteur d'un texte intitulé Messias puer, considéré comme étant perdu par l'ensemble de la recherche actuelle. Le présent article entend faire la démonstration que l'ouvrage catalogué sous le titre Historice evangelica initium est en réalité le Messias puer de Knorr. Nous en donnons ici une première description qui se veut programmatique en faisant état des nouvelles questions qui émergent suite à cette importante découverte.

\footnotetext{
${ }^{39}$ À travers, notamment, une référence récurrente à la sefira Yesod qui désigne couramment le sexe masculin dans la tradition kabbalistique.
} 


\section{BIBLIOGRAPHIE}

ARNOLD 2009: W. ARNOLD, «Jacob Burckhard aus Sulzbach als Bibliothekar in Wolfenbüttel », Morgen-Glantz : Zeitschrift der Christian Knorr von Rosenroth-Gesellschaft, 19, p. 53-69.

BAYle 1687 : P. BAYle, Nouvelles de la République des Lettres. Mois de janvier 1687, Henry Desbordes, Amsterdam.

BiAle 2001 : D. BIALE, Gershom Scholem: cabale et contre-histoire, Éditions de l'éclat, Paris, 2001.

Burnett 2000 : S.G. BurnetT, « Christian Hebrew Printing in the Sixteenth Century: Printers, Humanism and the Impact of the Reformation », Helmantica: Revista de Filología Clásica y Hebrea, 51, n 154, p. 13-42.

COUDERT 1995 : A.P. COUDERT, Leibniz and the Kabbalah, Springer, Dordrecht.

COUdERT 1996 : A.P. COUdERT, « Leibniz et Christian Knorr von Rosenroth : une amitié méconnue », Revue de l'histoire des religions, 213, n 4, p. 467-484.

DeUtSCHE AKADEMIE DER WisSENSCHAFTEN 1923: Gottfried Wilhelm Leibniz: Sämtliche Schriften und Briefe, Deutsche Akademie der Wissenschaften, Darmstadt und Berlin, Akademie-Verlag.

GAREL 2018: M. GAREL, « Knorr von Rosenroth Christian (1636-1689)», dans Encyclopcedia Universalis [en ligne], http://www.universalis.fr/encyclopedie/christianknorr-von-rosenroth/. Consulté le 22 février 2018.

KNORR DE RoSEnROTH 1677 : C.K. de RosenRoth, Kabbala denudata I, Sulzbaci.

Knorr De Rosenroth 1684a : C.K. de Rosenroth, דיתיקא חדתא Novum Domini Nostri Jesu Christi Testamentum Syriace, Sulzbaci.

KNORR DE Rosenroth 1684b : C.K. de Rosenroth, Kabbala denudata, II, Francofurti.

KNORR DE RoSENROTH 1684c : C. K. de RosenRoth, Liber Sohar, Sultzbaci.

KnORR De Rosenroth 1689 : C. K. de Rosenroth, Historice evangelicae initium, Cod. Guelf. 126 Extrav., Herzog August Bibliothek.

Ludolf 1681 : H. Ludolf, Historia Aethiopica, sive brevis et succincta descriptio regni Habessinorum, Francofurti ad Moenum, Joh. Dav. Zunner.

MORERI 1740 : L. MORERI, Le grand dictionnaire historique ou Le mélange curieux de l'histoire sacrée et profane, chez Brunel, Amsterdam.

ROUSSE-LACORDAIRE 2018 : Esquisse de la kabbale chrétienne, introduit, traduit et annoté par RousSe-LACORDAIRE, J., Les Belles Lettres, Paris.

SCHOLEM 1988a : G. SCHOLEM, «L'étude de la kabbale, depuis Reuchlin, jusqu'à nos jours », dans Le Nom et les symboles de Dieu dans la mystique juive, Cerf, Paris, p. 191-203.

SCHOLEM 1988b : G. SCHOLEM, «Exposé des motifs véritables qui m'incitèrent à étudier la kabbale », dans Le Nom et les symboles de Dieu dans la mystique juive, Cerf, Paris, p. 7-9.

SCHOLEM 1990 : G. SCHOLEM, De la création du monde à Varsovie, Cerf, Paris [1984¹]

VAN GEMERT 1991: G. VAN GEMERT, «Frühe niederländische Stimmen zu Christian Knorr von Rosenroth und ihr Kontext Knorr-Artikel in Lexiken aus der ersten Hälfte des 18. 
Jahrhunderts », Morgen-Glantz: Zeitschrift der Christian Knorr von RosenrothGesellschaft, 1, p. 79-90.

Vileno 2016 : A.M. VILENO, À l'ombre de la kabbale. Philologie et ésotérisme au XVII siècle dans l'œuvre de Knorr de Rosenroth, Honoré Champion, Paris.

ZELler 2006: R. ZELLER, «Der Nachlass Christian Knorr von Rosenroth in der Herzog August Bibliothek in Wolfenbüttel », Morgen-Glantz : Zeitschrift der Christian Knorr von Rosenroth-Gesellschaft, 16, p. 55-71. 
